\title{
Clinical Outcomes and Healthcare Resource Utilization in a Real-World Population Reflecting the DAPA-CKD Trial Participants
}

\author{
Tope Olufade (D) · Lois Lamerato · Juan José García Sánchez • \\ Like Jiang · Joanna Huang · Stephen Nolan · Janani Rangaswami
}

Received: November 16, 2020 / Accepted: December 11, 2020 / Published online: January 20, 2021

(C) The Author(s) 2021

\begin{abstract}
Introduction: The DAPA-CKD trial assessed dapagliflozin in patients with chronic kidney disease (CKD) with or without type 2 diabetes (T2D). To aid interpretation of results, renal and cardiovascular outcomes plus healthcare resource utilization (HCRU) and costs were assessed in a real-world population similar to that of DAPA-CKD.

Methods: Henry Ford Health System (2006-2016) data were used to identify patients with CKD stages 2-4 [estimated glomerular filtration rate (eGFR) $25-75 \mathrm{ml} / \mathrm{min} / 1.73 \mathrm{~m}^{2}$ at index and urine albumin-to-creatinine ratio (UACR) $0-5000 \mathrm{mg} / \mathrm{g} ; \quad n=22,251]$. Included patients had confirmatory eGFR $\geq 90$ days postindex and no kidney transplant or progression
\end{abstract}

Supplementary Information The online version contains supplementary material available at https:// doi.org/10.1007/s12325-020-01609-2.

T. Olufade $(\bowtie) \cdot$ L. Jiang $\cdot$ J. Huang

AstraZeneca, Wilmington, DE, USA

e-mail: Tope.Olufade@astrazeneca.com

L. Lamerato

Department of Public Health Sciences, Henry Ford

Health System, Detroit, MI, USA

J. J. G. Sánchez · S. Nolan

AstraZeneca, Cambridge, UK

J. Rangaswami

Einstein Medical Center, Philadelphia, PA, USA to end-stage kidney disease during 12 months pre-index. The final population $(n=6557)$ was stratified by UACR (0-29, 30-199 and 200-5000 mg/g; the last comprising the DAPACKD-like cohort). Patients were followed for 5 years post-index.

Results: Adverse clinical outcomes incidence increased with UACR and was highest for the DAPA-CKD-like cohort (UACR 200-5000 mg/g) versus lower UACR categories $(0-29 \mathrm{mg} / \mathrm{g}$ and 30-199 mg/g): renal composite outcome (progression to CKD stage 5, dialysis, transplant, $\geq 50 \%$ sustained eGFR decline): $26.0 \%$ versus $2.2 \%$ and $5.8 \%$; heart failure (HF): $36.1 \%$ versus $13.9 \%$ and $24.6 \%$; myocardial infarction: $11.3 \%$ versus $4.7 \%$ and $7.4 \%$; stroke: $8.9 \%$ versus $4.0 \%$ and $5.7 \%$; and mortality: $18.5 \%$ versus $6.0 \%$ and $11.7 \%$, respectively. Within the DAPA-CKD-like cohort, patients with versus without T2D or HF had a higher frequency of adverse outcomes. The DAPA-CKD-like cohort also had significantly higher annualized perpatient healthcare costs $(\$ 39,222 /$ year versus $\$ 19,547 /$ year), hospital admission rate (0.55/ year versus 0.20 /year) and outpatient specialist visit rate $(7.55 /$ year versus 6.74 /year) versus the lowest UACR category.

Conclusion: The significant adverse renal and cardiovascular outcomes observed, particularly in the DAPA-CKD-like cohort, represent a substantial burden resulting in increased mortality, HCRU and costs, demonstrating the need for additional treatment options. 
Keywords: Albuminuria; Chronic kidney disease; Dapagliflozin; Healthcare resource utilization; Heart failure; Real-world outcomes; Type 2 diabetes

\section{Key Summary Points}

The DAPA-CKD trial assessed dapagliflozin in patients with chronic kidney disease (CKD) with and without type 2 diabetes.

To aid interpretation of the DAPA-CKD results, renal and cardiovascular outcomes, plus healthcare resource utilization (HCRU) and costs, were assessed in a real-world population reflective of the DAPA-CKD trial.

Incidence of adverse clinical outcomes (overall renal composite outcome, heart failure, myocardial infarction, stroke, mortality) increased with UACR and was highest for the DAPA-CKD-like cohort vs. lower UACR categories.

The DAPA-CKD-like cohort also had significantly higher annualized perpatient healthcare costs, rates of hospital admission and rates of outpatient specialist visits vs. the lowest UACR category.

The significant adverse renal and cardiovascular outcomes observed, particularly in the DAPA-CKD-like cohort, represent a substantial burden resulting in increased mortality and HCRU and costs, demonstrating the need for additional treatment options.

\section{DIGITAL FEATURES}

This article is published with digital features, including a summary slide, to facilitate understanding of the article. To view digital features for this article go to https://doi.org/10.6084/ m9.figshare.13365545.

\section{INTRODUCTION}

Chronic kidney disease (CKD), defined as either abnormalities of kidney structure marked by damage or decreased function indicated by declining estimated glomerular filtration rate (eGFR) present for $>3$ months, is a worldwide public health problem [1]. The classification of CKD is based on etiology, eGFR and urine albumin-to-creatinine ratio (UACR). Patients are more commonly categorized by eGFR, ranging from stage 1 (eGFR $\geq 90 \mathrm{ml} / \mathrm{min} /$ $1.73 \mathrm{~m}^{2}$ ), with mild kidney damage, to stage 5 (eGFR $<15 \mathrm{ml} / \mathrm{min} / 1.73 \mathrm{~m}^{2}$ ), signifying kidney failure and usually a need for dialysis or kidney transplant; however, guideline recommendations state that a patient's eGFR category should be combined with UACR to aid staging and risk stratification. UACR is an important predictor of risk for disease progression, adverse outcomes and mortality $[1,2]$. Categories range from A1 (UACR $<30 \mathrm{mg} / \mathrm{g}$ ) to normal/mildly increased, to A3 (UACR $>300 \mathrm{mg} / \mathrm{g}$ ) and severely increased [1].

Between 2013 and 2016, an estimated 14.8\% of the US adult population had CKD (stages 1-5), with CKD stage 3 (6.4\%) being the most common [3]. Patients with CKD are at a significantly increased risk of adverse clinical outcomes, including cardiovascular events (e.g. heart failure $[\mathrm{HF}])$, hospitalization, end-stage kidney disease (ESKD) and premature death, which are directly associated with the degree of CKD severity $[1,4,5]$. CKD is also associated with substantial mortality, morbidity and healthcare costs. Medicare spending (excluding dialysis) for all beneficiaries who had CKD (12.5\%) exceeded $\$ 84$ billion in 2017, an increase of $6.3 \%$ from 2016 [6]. After combining an additional $\$ 36$ billion attributable to ESKD costs, total Medicare spending on both CKD and ESKD was over $\$ 120$ billion, representing $33.8 \%$ of the total Medicare fee-for-service spending [6].

Management of risk factors for CKD progression (e.g., systolic blood pressure and glycated hemoglobin in patients with diabetes) is key to the treatment of patients with CKD; however, there remains a lack of dedicated 
evidence-based treatment options that slow disease progression and improve patient outcomes. Trials of renin-angiotensin-aldosterone system (RAAS) inhibitors have demonstrated reno-protective effects, such as a reduction in the risk of doubling of serum creatinine and the development of ESKD in patients with type 2 diabetes (T2D) and nephropathy [7-9], although evidence in patients without T2D has been scarce [10]. There has been a lack of success in developing newer therapies on top of RAAS inhibitors as current standard of care to delay progression and improve outcomes in patients with or without T2D.

Sodium-glucose co-transporter 2 inhibitors have demonstrated significant cardiorenal benefits in previous trials in patients with T2D $[11,12]$ as well as cardiovascular benefits in patients with HF with reduced ejection fraction in the presence or absence of diabetes [13-15]. In the Dapagliflozin and Prevention of Adverse Outcomes in CKD (DAPA-CKD) trial, which assessed the effect of dapagliflozin on kidney outcomes and mortality in patients with CKD and elevated UACR (200-5000 mg/g), dapagliflozin significantly reduced the composite of risk of CKD progression, ESKD, renal or cardiovascular death (hazard ratio [HR], 0.61 [95\% confidence interval [CI] 0.51-0.72]), cardiovascular death or HF hospitalization (HR, 0.71 [95\% CI 0.55-0.92]) and all-cause mortality (HR, 0.69 [95\% CI 0.53-0.88]) in patients with or without T2D [16].

To understand the CKD disease burden at a population level in a routine clinical practice setting, and to aid clinical implementation of the DAPA-CKD results, we report real-world clinical outcomes and healthcare resource utilization (HCRU) from the Henry Ford Health System (HFHS) in a patient population that meets the DAPA-CKD trial criteria and compare these to patients with similar CKD staging but lower UACR.

\section{METHODS}

\section{Study Design}

This was a retrospective, longitudinal, observational study evaluating information from electronic healthcare records (EHRs) and an administrative data set of the HFHS. The HFHS is a large integrated US healthcare delivery system that provides almost 950,000 patients with care annually, with 4.2 million outpatient encounters, 100,000 surgeries and 113,000 hospitalizations. The HFHS operates in five regions throughout South-East Michigan, providing data that can be utilized to examine realworld outcomes of patients with CKD across different populations, facilitating the interpretation and practical implications of clinical studies [17]. The study was approved by the HFHS Institutional Review Board.

\section{Patients}

Patients from HFHS data (2006-2016) were identified with CKD stages 2-4 (eGFR 25-75 ml/ $\min / 1.73 \mathrm{~m}^{2}$, calculated using the Chronic Kidney Disease Epidemiology Collaboration [CKD-EPI] equation) and UACR (0-5000 mg/g). The index event was the first qualifying eGFR. A second eGFR $\geq 90$ days after index was used to confirm chronicity of the disease and to rule out acute kidney injury. Additional inclusion criteria included being $\geq 18$ years old and having $\geq 1$ UACR measurement $(0-5000 \mathrm{mg} / \mathrm{g})$ within 6 months pre- and post-index. Exclusion criteria included: history of kidney transplant or dialysis any time pre-index date or within 6 months post-index date, progression to CKD stage 5 within 6 months of the index date, no UACR measurement or UACR $>5000 \mathrm{mg} / \mathrm{g}$ during baseline (the 12 months pre-index), and any history or diagnosis 6 months post index of type 1 diabetes, polycystic kidney disease, lupus nephritis or anti-neutrophil cytoplasmic antibody nephritis. Each patient was followed for 5 years after the index date. The study timeline is presented in Fig. 1. 


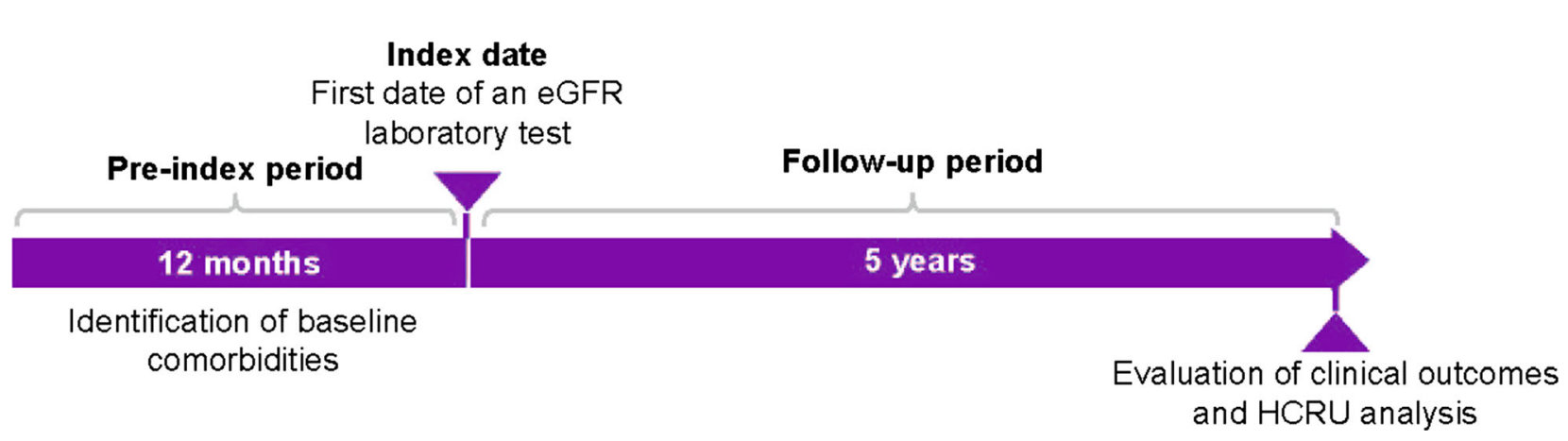

Fig. 1 Study timeline. eGFR estimated glomerular filtration rate, HCRU healthcare resource utilization

\section{Assessments}

Primary outcomes were renal composite outcomes, which consisted of progression to CKD stage 5, onset of dialysis, kidney transplant and/ or $\geq 50 \%$ sustained decline in eGFR (second $\geq 50 \%$ decline measured at least 28 days apart) as well as cardiovascular outcomes and mortality. Cardiovascular outcomes were comprised of myocardial infarction (MI), hospitalization for MI, stroke, hospitalization for stroke, HF, and hospitalization for HF. Any hospitalizations for the given outcome during the 5 years of follow-up were counted. All-cause mortality during the follow-up period was also investigated, and all clinical outcomes were evaluated for up to 5 years follow-up (cumulative).

Secondary outcomes included HCRU and costs, comprising all-cause hospital admissions, inpatient days, emergency department visits, outpatient primary care visits, outpatient specialist visits and total outpatient visits. Data extracted from the administrative database were coded specifically for each of these encounter types. Outcomes were evaluated by rate/year, except inpatient days, which were calculated by length of stay. Cumulative HCRU outcomes were based on follow-up time. Patients were censored on date of death, last contact with the health system or on December 31, 2018. Allcause costs of care were annualized charges. Billing data on all patient charges were used as proxy for costs of care; medication costs were not available.

Patients were stratified into three cohorts by UACR: $\quad 0-29 \mathrm{mg} / \mathrm{g}, \quad 30-199 \mathrm{mg} / \mathrm{g}$ and
200-5000 $\mathrm{mg} / \mathrm{g}$, the last comprising the DAPACKD-like cohort. Analyses in the DAPA-CKDlike cohort were additionally stratified by baseline HF and T2D. Outcomes were additionally assessed by eGFR and UACR categories.

\section{Statistical Analysis}

Categorical variables were presented as count and percentage of patients in each category; continuous variables were summarized by providing the means and standard deviations. Descriptive statistics provided the overall characteristics of the study population at baseline. Chi-square statistics were used to compare the clinical outcomes in the three study cohorts. Analysis of variance was used to compare the mean HCRU outcomes between groups, based on annualized utilization using the duration of follow-up for each patient.

\section{RESULTS}

\section{Patients}

The source population included 22,251 patients; applying the eligibility criteria resulted in a final study population of 6557 (Fig. 2). Notably, 9702 of 22,251 (43.6\%) patients meeting the eGFR criteria were excluded from the study as they had no available UACR measurement. Patient baseline characteristics and demographics are presented in Table 1. Notably, the DAPA-CKD-like cohort (UACR $200-5000 \mathrm{mg} / \mathrm{g}$ ) had the highest baseline burden of comorbid HF and history of 


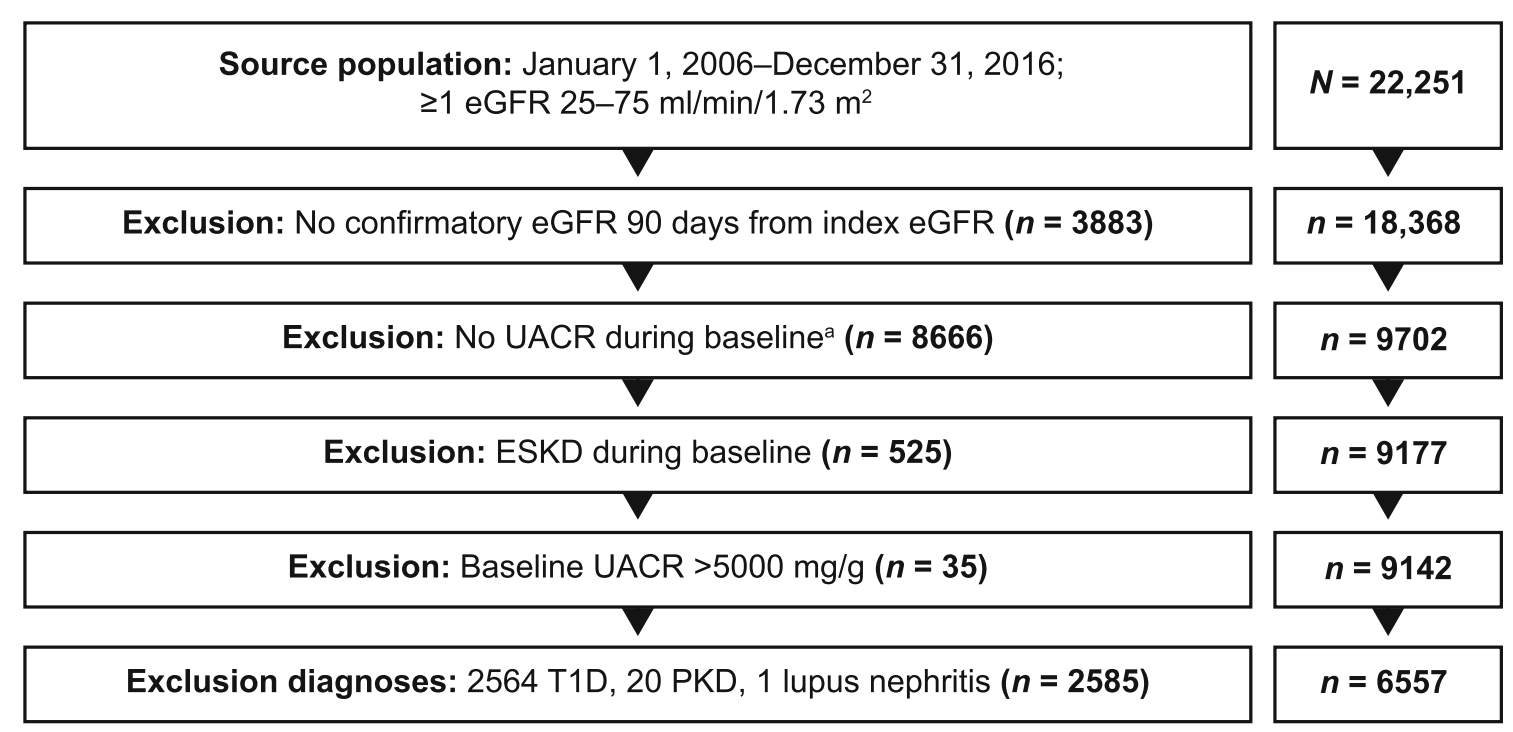

Fig. 2 Patient attrition diagram. eGFR estimated glomerular filtration rate, ESKD end-stage kidney disease, $P K D$ polycystic

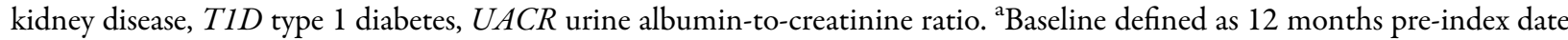

cardiovascular events. Baseline characteristics according to T2D status and HF are shown in Table S1 and Table S2, respectively.

\section{Clinical Outcomes at 5-Year Follow-up}

Across all adverse clinical outcomes assessed, increased risk was observed in patients with increased UACR, with the highest incidence in the DAPA-CKD-like cohort versus patients in lower UACR categories (Fig. 3a); notable outcomes included the overall renal composite outcome $(26.0 \%$ in the DAPA-CKD-like cohort vs. $5.8 \%$ in the UACR $30-199 \mathrm{mg} / \mathrm{g}$ category and $2.2 \%$ in the UACR $0-29 \mathrm{mg} / \mathrm{g}$ category) and all-cause mortality $(18.5 \%$ in the DAPA-CKDlike cohort vs. $11.7 \%$ in the UACR $30-199 \mathrm{mg} / \mathrm{g}$ category and $6.0 \%$ in the UACR $0-29 \mathrm{mg} / \mathrm{g}$ category). Within the DAPA-CKD-like cohort, patients with T2D or HF had a numerically higher frequency of adverse outcomes than patients without these comorbidities. There were no statistical differences between those with and without T2D. Patients with HF had statistically increased incidences of the overall renal composite, MI and all-cause mortality outcomes compared to patients without HF (Fig. 3b, c).

\section{Healthcare Resource Utilization and Costs at 5-Year Follow-up}

Costs of care per patient in the DAPA-CKD-like cohort were significantly higher and more than double that of the lowest UACR category (UACR $0-29 \mathrm{mg} / \mathrm{g})$, at $\$ 39,222 /$ year versus $\$ 19,547 /$ year, respectively (Fig. 4a). Hospital admission rates for patients in the DAPA-CKD-like cohort were almost three times higher than those for patients in the lowest UACR category $(0.55 /$ year vs. 0.20/year, respectively; Fig. 4a). Additionally, length of stay was over 3 times higher in the DAPA-CKD-like cohort than in the lowest UACR category (Fig. 4a).

In the DAPA-CKD-like cohort, HCRU and costs were comparable between patients with and without T2D (Fig. 4b). However, patients with baseline HF had significantly increased acute and ambulatory care needs and costs versus patients without HF (Fig. 4c). This burden is already evident in the early stages of CKD; more granular outputs according to the Kidney Disease: Improving Global Outcomes 2012 categorization are reported in the supplementary material (Tables S3 and S4). Overall, costs increased across the ascending UACR categories (from 0-29 mg/g to $30-199 \mathrm{mg} / \mathrm{g}$ up to $200-5000 \mathrm{mg} / \mathrm{g}$ ). This was seen both for the 
Table 1 Baseline demographics ${ }^{a}$ and characteristics stratified by UACR category

\begin{tabular}{|c|c|c|c|c|c|}
\hline UACR category $(\mathrm{mg} / \mathrm{g})$ & $0-29(n=4331)^{\mathrm{b}}$ & $30-199(n=1354)^{\mathrm{b}}$ & $200-5000(n=872)^{\mathrm{b}}$ & Total $(N=6557)^{c}$ & $P$ value \\
\hline \multicolumn{6}{|l|}{ Gender, $n(\%)$} \\
\hline Male & $1885(62.2)$ & $679(22.4)$ & $466(15.4)$ & $3030(46.2)$ & $<0.0001$ \\
\hline Female & $2446(69.4)$ & $675(19.1)$ & $406(11.5)$ & $3527(53.8)$ & \\
\hline Age (years), mean (SD) & $63.1(8.2)$ & $63.3(8.7)$ & $61.4(9.4)$ & $62.9(8.5)$ & $<0.0001$ \\
\hline \multicolumn{6}{|l|}{ CKD stage, $n(\%)^{\mathrm{d}}$} \\
\hline 2 & $2691(73.2)$ & $676(18.4)$ & $309(8.4)$ & $3676(56.1)$ & $<0.0001$ \\
\hline $3 a$ & $1275(62.6)$ & $466(22.9)$ & $297(14.6)$ & $2038(31.1)$ & \\
\hline $3 b$ & $337(45.7)$ & $186(25.2)$ & $215(29.1)$ & $738(11.3)$ & \\
\hline 4 & $28(27.7)$ & $26(24.8)$ & $51(48.6)$ & $105(1.6)$ & \\
\hline $\mathrm{eGFR}\left(\mathrm{ml} / \mathrm{min} / 1.73 \mathrm{~m}^{2}\right)$, mean $(\mathrm{SD})$ & $61.5(10.7)$ & $58.0(12.3)$ & $52.8(14.2)$ & $59.6(11.9)$ & $<0.0001$ \\
\hline UACR $(\mathrm{mg} / \mathrm{g})$, mean $(\mathrm{SD})$ & $10.5(6.8)$ & $76.8(41.3)$ & $1086.8(1040.5)$ & $167.3(524)$ & $<0.0001$ \\
\hline Serum potassium, $n$ & 3966 & 1245 & 801 & 6012 & \\
\hline mmol/l, mean $(\mathrm{SD})$ & $4.3(0.4)$ & $4.4(0.5)$ & $4.4(0.5)$ & $4.3(0.4)$ & $<0.05$ \\
\hline HbAlc, $n$ & 3036 & 916 & 516 & 4468 & \\
\hline$\%$, mean $(\mathrm{SD})$ & $7.3(1.4)$ & $7.8(1.8)$ & $8.2(2.0)$ & $7.5(1.6)$ & $<0.001$ \\
\hline \multicolumn{6}{|l|}{ Comorbidities, $n(\%)^{\mathrm{e}}$} \\
\hline $\mathrm{T} 2 \mathrm{D}$ & $3828(88.4)$ & $1196(88.3)$ & $752(86.2)$ & $5776(88.1)$ & NS \\
\hline Heart failure & $225(5.2)$ & $120(8.9)$ & $122(14.0)$ & $467(7.1)$ & $<0.001$ \\
\hline $\mathrm{T} 2 \mathrm{D}$ and heart failure & $205(4.7)$ & $115(8.5)$ & $115(13.2)$ & $435(6.6)$ & $<0.001$ \\
\hline Hypertension & $3130(72.3)$ & $987(72.9)$ & $670(76.8)$ & $4787(73.0)$ & $<0.05$ \\
\hline Hypertensive nephropathy & $156(3.6)$ & $111(8.2)$ & $152(17.4)$ & $419(6.4)$ & $<0.001$ \\
\hline Glomerulonephritis & $11(0.3)$ & $7(0.5)$ & $25(2.9)$ & $43(0.7)$ & $<0.001$ \\
\hline Myocardial infarction & $53(1.2)$ & $29(2.1)$ & $33(3.8)$ & $115(1.8)$ & $<0.001$ \\
\hline Stroke & $45(1.0)$ & $18(1.3)$ & $21(2.4)$ & $84(1.3)$ & $<0.01$ \\
\hline Peripheral artery disease & $152(3.5)$ & $71(5.2)$ & $59(6.8)$ & $282(4.3)$ & $<0.001$ \\
\hline Coronary artery disease & $533(12.3)$ & $174(12.9)$ & $127(14.6)$ & $834(12.7)$ & NS \\
\hline Dyslipidemia & $2513(58.0)$ & $723(53.4)$ & $441(50.6)$ & $3677(56.1)$ & $<0.001$ \\
\hline
\end{tabular}

CKD chronic kidney disease, $e$ GFR estimated glomerular filtration rate, $H b A 1 c$ glycated hemoglobin, $N S$ not significant, $S D$ standard deviation, $T 2 D$ type 2 diabetes, $U A C R$ urine albumin-to-creatinine ratio

${ }^{\text {a }}$ Baseline defined as 12 months pre-index date

b Percentages calculated using the total for that row as denominator

c Percentages calculated using the total for that column

d CKD stages 2, 3a, 3b and 4: eGFR 60-75, 45 to $<60,30$ to $<45$ and 25 to $<30 \mathrm{ml} / \mathrm{min} / 1.73 \mathrm{~m}^{2}$, respectively

${ }^{\mathrm{c}}$ Comorbidities based on diagnoses codes for clinical encounters. Significance calculated using chi-square analysis for categorical variables and analysis of variance for mean age

overall UACR groups (Fig. 4a) and when patients were categorized further by eGFR category; costs also increased with declining eGFR level (Table S4A). An exception was the highest UACR category studied (200-5000 mg/g), where costs were lower in the subgroup of patients with eGFR $25-<30 \mathrm{ml} / \mathrm{min} / 1.73 \mathrm{~m}^{2}$, although patient numbers were relatively low for this eGFR category (Table S4A).

\section{DISCUSSION}

In this study, a large population of patients with CKD was stratified into three cohorts by UACR, with the highest range $(200-5000 \mathrm{mg} / \mathrm{g})$ comprising the DAPA-CKD-like cohort. The incidences of adverse renal, cardiovascular and mortality outcomes were high overall, but were highest in the DAPA-CKD-like cohort compared 
with patients in lower UACR categories; this was seen in both patients with and without T2D as well as those with and without HF. HCRU and costs were also higher in the DAPA-CKD-like cohort compared with the lower UACR categories. The impact of UACR category on HCRU and costs of care has not been previously reported for patients with CKD.

Previous renal outcome trials in CKD have failed to demonstrate a significant benefit from the tested therapeutic intervention in all-cause mortality, despite a reduction in CKD progression $[8,9,12,18]$. The overall renal composite outcome and all-cause mortality in this study ( $26.0 \%$ and $18.5 \%$, respectively) were notably increased in the DAPA-CKD-like cohort versus patients with lower UACR. HCRU and costs of care increased with UACR category, particularly in acute care. While this increase was seen with disease progression, there was a high clinical burden even at the early stages of CKD, suggesting a need for early intervention to slow disease progression.

These results highlight the current unmet need in a contemporary real-world population stratified by UACR, particularly for the cohort with UACR reflective of the DAPA-CKD trial inclusion criteria. However, widespread undertesting of UACR limits appropriate risk stratification of patients with CKD, which could result in delaying intervention or treatment. Low UACR testing in patients with CKD is not unique to the HFHS database; in a cohort study of EHRs from two large US healthcare systems (the Center for Kidney Disease Research, Education, and Hope [CURE-CKD] registry), approximately $90 \%$ of high-risk patients with CKD who should have been tested did not have UACR laboratory measurements available [19].

\section{Strengths}

The major strengths of this study include the large and diverse study population and the extensive follow-up period. The HFHS database used in this analysis captures high-quality data via its EHR, including a broad spectrum of relevant laboratory test results and longitudinal datapoints. Inputs made at the point of care are stored as structured data fields, and patients have a lifetime medical record number that is used across all content areas. All outpatient, emergency room, and inpatient encounters and procedures are captured using International Classification of Diseases 9th or 10th revision codes, and Current Procedural Terminology version 4 procedural codes. The strength of the database is also shown by the numerous publications from HFHS, including in journals such as Cancer Epidemiology, Biomarkers and Prevention, Journal of Obstetrics and Gynecology Canada and the American Journal of Otolaryngology.

UACR is not captured or reported well in many databases and as a result patient outcomes stratified by UACR category are often not available in the literature. By contrast, the source population utilized in this study included a high percentage of patients with UACR data compared with other studies, such as the study by Tuttle et al. [19]. The present study adds to the literature surrounding the clinical and financial burden associated with different UACR categories and aids in the understanding of the potential role that new therapies can play in addressing the residual risk in patients with CKD managed in a real-world setting.

The importance of UACR to consider as an additional risk marker as well as eGFR in patients with CKD can be shown by the findings of the present study. Although the percentage of patients with UACR in the highest risk category $(200-5000 \mathrm{mg} / \mathrm{g}$ ) was higher in the later CKD stages versus the earlier stages, there were still $8.4 \%$ of patients with CKD stage 2 and $14.6 \%$ in the highest UACR category. Similarly, in the DAPA-CKD trial (all patients with UACR $200-5000 \mathrm{mg} / \mathrm{g}$ at baseline), although the majority of patients had in the lowest ranges studied, $10.9 \%$ and $10.2 \%$ of patients in the dapagliflozin and placebo groups, respectively, had eGFR in the highest range studied (60-75 $\mathrm{ml} / \mathrm{min} / 1.73 \mathrm{~m}^{2}$, roughly equivalent to CKD stage 2) [16].

\section{Limitations}

This is a study based on real-world data that were collected observationally in the usual 


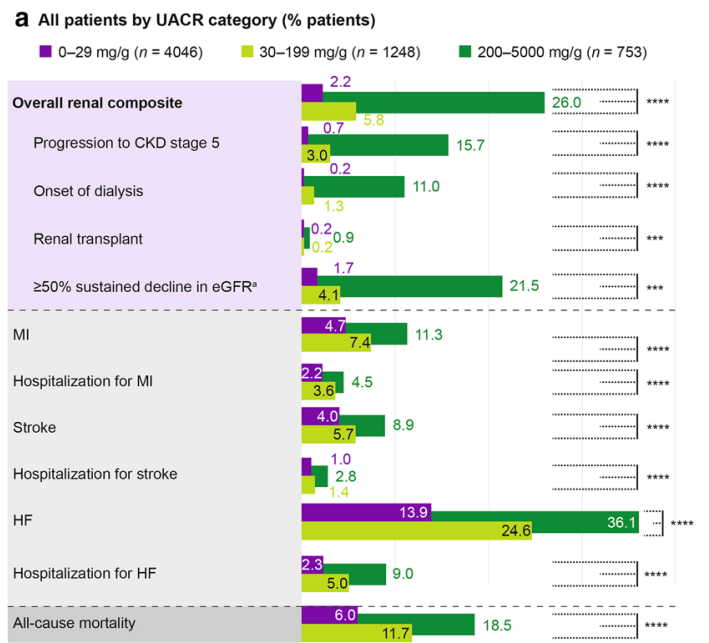

b DAPA-CKD-like population (eGFR $25-75 \mathrm{ml} / \mathrm{min} / 1.73 \mathrm{~m}^{2}$ and UACR $200-5000 \mathrm{mg} / \mathrm{g}$ ) by baseline T2D (\% patients)

Without T2D $(n=107) \quad$ With T2D $(n=646)$

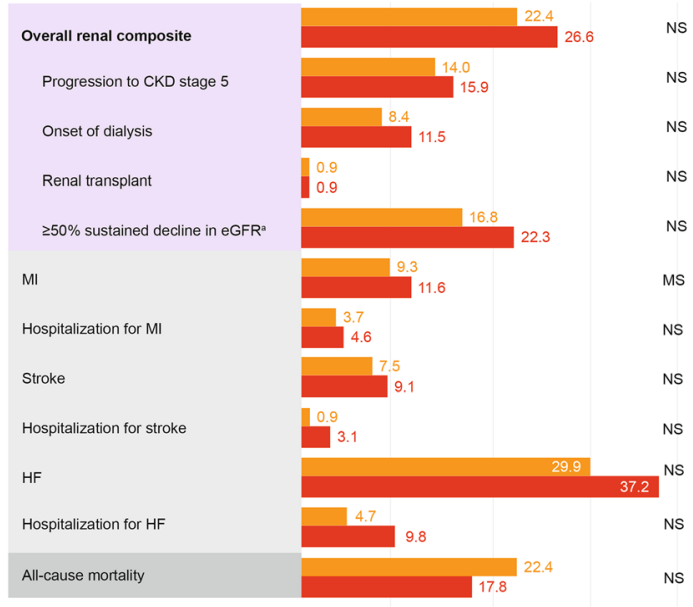

C DAPA-CKD-like population (eGFR 25-75 $\mathrm{ml} / \mathrm{min} / 1.73 \mathrm{~m}^{2}$ and UACR $200-5000 \mathrm{mg} / \mathrm{g}$ ) by baseline $\mathrm{HF}$ (\% patients)

- Without HF $(n=648) \quad$ With HF $(n=105)$

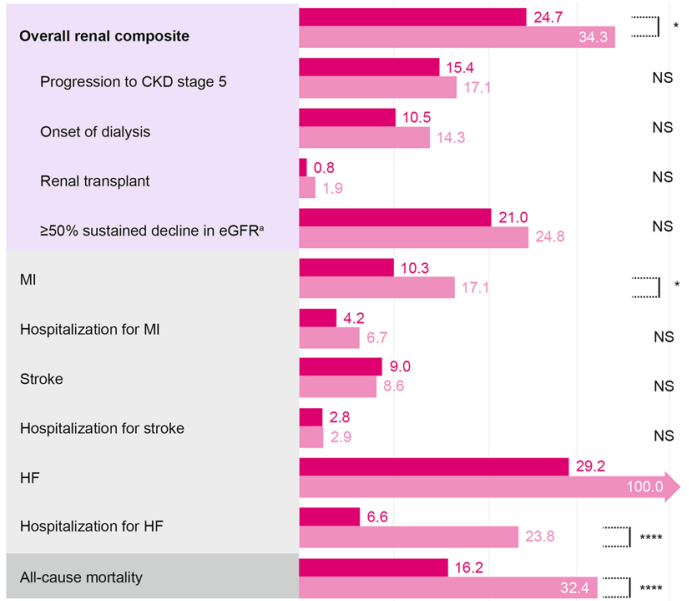

4 Fig. 3 Clinical outcomes at 5 years. $C K D$ chronic kidney disease, eGFR estimated glomerular filtration rate, $H F$ heart failure, $M I$ myocardial infarction, $N S$ not significant, $T 2 D$ type 2 diabetes, $U A C R$ urine albumin-to-creatinine ratio. ${ }^{a}$ Second eGFR $>50 \%$ decline at least 28 days apart. Cardiac outcomes (MI, stroke and HF) include patients with any encounter during 5 years of follow-up. Hospitalization outcomes include patients with a hospitalization for the given outcome during 5 years of follow-up. Statistical significance of patient outcomes calculated using chi-square analysis. ${ }^{*} P<0.05,{ }^{* *} P<0.01,{ }^{* * *} P<0.001$, ${ }^{* * * *} P<0.0001$. Axes cropped at $40 \%$ to enhance visual assessment. Patients were excluded if they had no eGFR or clinical encounter in the fifth year of follow-up

clinical setting through electronic medical records or administrative claims, in contrast to the clinical trial setting. The DAPA-CKD trial composite primary outcome component of death from cardiovascular and renal causes could not be analyzed in the present study population due to lack of data availability; however, all-cause mortality was investigated. Comprehensive assessment of adherence to standard of care was also not possible because of the lack of availability of baseline medication status. Although the cost of medications was not available, we reported on healthcare costs stratified by UACR measurement, which have not previously been reported in the literature. Findings of this study are based on a single healthcare system and so may not be generalizable to the entire US population; additionally, HCRU and associated costs of care may differ by region and healthcare systems within the US and compared with Medicare. Although utilization of HCRU outside of the health care system was not available for this study, the patients in this analysis had long-term care within the health system; hence, most of the HCRU is likely captured. Additionally, administrative data are subject to potential coding error and are not collected for research.

The majority of the study population had T2D (88.1\%), which may limit comparisons to previous studies of patients with CKD that had higher proportions of patients without diabetes [2]. Although this may be reflective of clinical 


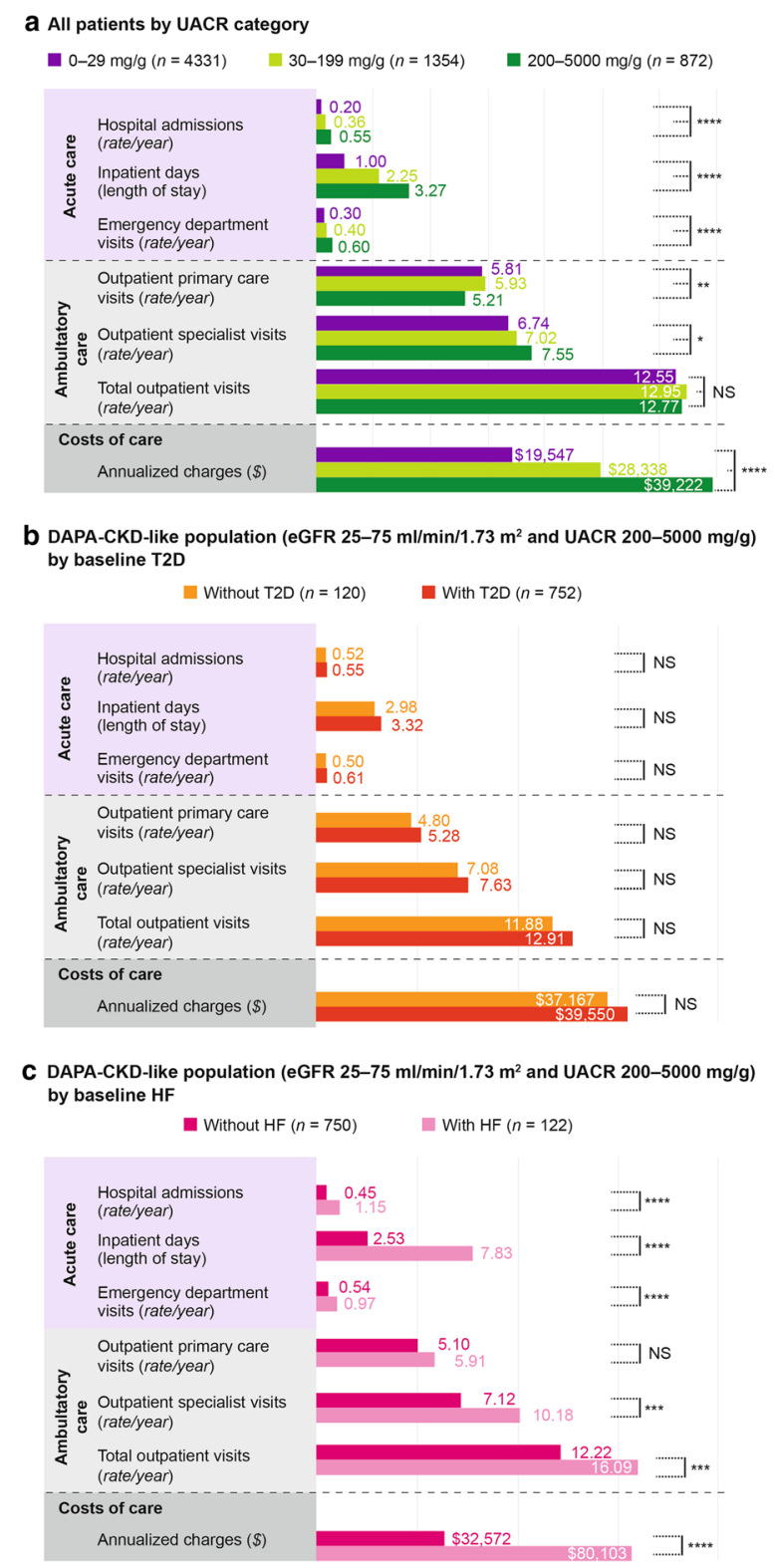

Fig. 4 Healthcare resource utilization and costs. eGFR estimated glomerular filtration rate, $H F$ heart failure, $N S$ not significant, T2D type 2 diabetes, $U A C R$ urine albumin-to-creatinine ratio. Utilization rates are the total observed utilization divided by follow-up time and reported as an annual rate. Healthcare resource utilization/costs cover the entire follow-up period. Annualized charges are per patient. ${ }^{*} P<0.05, \quad{ }^{* *} P<0.01$, ${ }^{* * *} P<0.001,{ }^{* * * *} P<0.0001$. Statistical significance calculated using analysis of variance

practice where, if done, UACR testing is more likely to be conducted in patients with T2D
[19], this may also limit applicability of results to the DAPA-CKD trial, which enrolled $32.5 \%$ of patients without T2D [20]. However, it should be noted that a trend for similar clinical and HCRU/cost outcomes between patients with and without T2D was observed in the present study.

As a selection criterion, UACR measurement may have enriched the study population with a higher burden of comorbidities due to the increased likelihood of UACR monitoring at baseline. In addition, a considerable proportion of the source population did not have a UACR measurement during baseline, an exclusion criterion that restricted the size of the final study population. The cohort without comorbid T2D or HF included in this study may not be fully representative of real-world patients with $\mathrm{CKD}$, but may simply be reflective of the population tested for UACR in clinical practice because of the limited UACR testing undertaken in the overall HFHS database/population. Given the importance of UACR in disease staging [1], and the association of UACR with adverse outcomes demonstrated in this analysis, more needs to be done to increase the frequency of UACR testing across all patients with and at risk of CKD.

\section{CONCLUSIONS}

CKD is associated with a high burden of disease, mortality and healthcare costs. This study aimed to aid clinical interpretation and implementation of the DAPA-CKD trial results by assessing the residual renal, cardiovascular and mortality risk, along with the HCRU and costs, in a contemporary real-world CKD population.

Results of this analysis demonstrated significant and increased adverse renal, cardiovascular and mortality outcomes among patients with CKD stages 2-4 with elevated UACR (the DAPA-CKD-like cohort) as well as increased HCRU and costs compared with those in lower UACR categories. This was seen in patients both with and without comorbid T2D or HF, although in general patients with T2D or HF had a numerically higher frequency of adverse outcomes than patients without these 
comorbidities. There were no statistical differences in clinical outcomes between those with and without T2D, and increased incidences between those with and without HF. Patients with elevated UACR and baseline HF had significant increases in annual rate of hospital admissions, length of hospital stay, rate of emergency department visits, rate of outpatient specialist visits, rate of total outpatient visits and annualized charges compared with patients without HF.

Along with a need for more standardized UACR testing, there remains a need for more proactive management and additional therapies addressing the substantial residual risks for patients with $\mathrm{CKD}$-particularly patients with elevated UACR, reflective of the DAPA-CKD trial cohort. This will help address the burden of CKD progression and its attendant adverse cardiovascular profile with escalating healthcare costs, as well as improve outcomes in the patient population with kidney disease. Full cost-effectiveness analysis based on the DAPACKD trial outcomes would be beneficial as future research and, together with the assessment of the residual risk presented here, would further aid in healthcare implementation planning.

\section{ACKNOWLEDGEMENTS}

Funding. This analysis and development of this manuscript (including the journal's Rapid Serve and Open Access fees) were supported by AstraZeneca.

Role of the Sponsor. The sponsor (AstraZeneca) was involved in the study design and collection, analysis and interpretation of data as well as data checking of information provided in the manuscript. Ultimate responsibility for opinions, conclusions and data interpretation lies with the authors.

Medical Writing Assistance. Medical writing support was provided by Jessica Men, PharmD, and Annie Macpherson, PhD, and editorial support was provided by Sinead
Stewart, all of Core, London, UK, supported by AstraZeneca according to Good Publication Practice guidelines (http://annals.org/aim/ article/2424869/good-publication-practicecommunicating-company-sponsored-medicalresearch-gpp3).

Authorship. All named authors meet the International Committee of Medical Journal Editors criteria for the authorship of this article and take responsibility for the integrity of the work as a whole. They have given their approval for this version to be published.

Disclosures. Tope Olufade, Juan José García Sánchez, Like Jiang, Joanna Huang and Stephen Nolan are employees and stockholders of AstraZeneca. Lois Lamerato is an employee of Henry Ford Health System, which has received research support from AstraZeneca. Janani Rangaswami has nothing to disclosure.

Compliance with Ethics Guidelines. This study involved existing administrative data without direct patient contact. A relational database containing all relevant tables was created. All analyses were based on this database. This study was approved by HFHS Institutional Review Board under expedited approval, with waiver of informed consent. The only risk for human subjects was that of privacy breach, and that risk has been minimized by the following: the study data conformed to minimum necessary standards, the data were stored in a password-protected secure computing environment with access limited to HFHS principal investigator and study programmer, person-level data were and will not be released outside of HFHS, and results were reported at the aggregate level.

Data Availability. The datasets generated during and/or analyzed during the current study are available upon reasonable request in accordance with AstraZeneca's data sharing policy described at https:// astrazenecagrouptrials.pharmacm.com/ST/ Submission/Disclosure.

Open Access. This article is licensed under a Creative Commons Attribution- 
NonCommercial 4.0 International License, which permits any non-commercial use, sharing, adaptation, distribution and reproduction in any medium or format, as long as you give appropriate credit to the original author(s) and the source, provide a link to the Creative Commons licence, and indicate if changes were made. The images or other third party material in this article are included in the article's Creative Commons licence, unless indicated otherwise in a credit line to the material. If material is not included in the article's Creative Commons licence and your intended use is not permitted by statutory regulation or exceeds the permitted use, you will need to obtain permission directly from the copyright holder. To view a copy of this licence, visit http:// creativecommons.org/licenses/by-nc/4.0/.

\section{REFERENCES}

1. KDIGO CKD Work Group. KDIGO 2012 clinical practice guideline for the evaluation and management of chronic kidney disease. Kidney Int. 2013;3: 5-14.

2. Denker M, Boyle S, Anderson AH, et al. Overview and summary of selected findings. Clin J Am Soc Nephrol. 2015;10:2073-83.

3. USRDS. CKD in the General Population. 2018 USRDS Annual Data Report. 2018;1:28.

4. Go AS, Chertow GM, Fan D, McCulloch CE, Hsu CY. Chronic kidney disease and the risks of death, cardiovascular events, and hospitalization. N Engl J Med. 2004;351:1296-305.

5. Sarnak MJ, Levey AS, Schoolwerth AC, et al. Kidney disease as a risk factor for development of cardiovascular disease: a statement from the American Heart Association Councils on Kidney in Cardiovascular Disease, High Blood Pressure Research, Clinical Cardiology, and Epidemiology and Prevention. Circulation. 2003;108:2154-69.

6. USRDS. USRDS 2019 Annual Data Report. 2019. https://www.usrds.org/annual-data-report/currentadr/. Accessed Aug 2020.

7. Sica DA, Bakris GL. Type 2 diabetes: RENAAL and IDNT-the emergence of new treatment options. J Clin Hypertens (Greenwich). 2002;4:52-7.
8. Brenner BM, Cooper ME, de Zeeuw D, et al. Effects of losartan on renal and cardiovascular outcomes in patients with type 2 diabetes and nephropathy. N Engl J Med. 2001;345:861-9.

9. Lewis EJ, Hunsicker LG, Clarke WR, et al. Renoprotective effect of the angiotensin-receptor antagonist irbesartan in patients with nephropathy due to type 2 diabetes. N Engl J Med. 2001;345:851-60.

10. GISEN Group (Gruppo Italiano di Studi Epidemiologici in Nefrologia). Randomised placebo-controlled trial of effect of ramipril on decline in glomerular filtration rate and risk of terminal renal failure in proteinuric, non-diabetic nephropathy. Lancet. 1997;349:1857-63.

11. Neuen BL, Young T, Heerspink HJL, et al. SGLT2 inhibitors for the prevention of kidney failure in patients with type 2 diabetes: a systematic review and meta-analysis. Lancet Diabetes Endocrinol. 2019;7:845-54.

12. Perkovic V, Jardine MJ, Neal B, et al. Canagliflozin and renal outcomes in type 2 diabetes and nephropathy. N Engl J Med. 2019;380:2295-306.

13. McMurray JJV, Solomon SD, Inzucchi SE, et al. Dapagliflozin in patients with heart failure and reduced ejection fraction. N Engl J Med. 2019;381: 1995-2008.

14. Packer M, Anker SD, Butler J, et al. Cardiovascular and renal outcomes with empagliflozin in heart failure. N Engl J Med. 2020;383:1413-24.

15. Kosiborod M, Cavender MA, Fu AZ, et al. Lower risk of heart failure and death in patients initiated on sodium-glucose cotransporter- 2 inhibitors versus other glucose-lowering drugs: the CVD-REAL study (comparative effectiveness of cardiovascular outcomes in new users of sodium-glucose cotransporter-2 inhibitors). Circulation. 2017;136:249-59.

16. Heerspink HJL, Stefansson BV, Correa-Rotter R, et al. Dapagliflozin in patients with chronic kidney disease. N Engl J Med. 2020;383:1436-46.

17. Henry Ford Health Systems. Fact Sheet. 2019. https://www.henryford.com/-/media/files/henryford/2018-fact-sheet-final.pdf?la=en. Accessed Aug 2020 .

18. Stengel B, Combe C, Jacquelinet C, et al. The French chronic kidney disease-renal epidemiology and information network (CKD-REIN) cohort study. Nephrol Dial Transpl. 2014;29:1500-7.

19. Tuttle K, Alicic R, Duru O, et al. Clinical characteristics of and risk factors for chronic kidney disease among adults and children: an analysis of the 
CURE-CKD Registry. JAMA Netw Open. 2019;2: e1918169.

20. Heerspink HJL, Stefansson BV, Chertow GM, et al. Rationale and protocol of the Dapagliflozin And
Prevention of Adverse outcomes in Chronic Kidney Disease (DAPA-CKD) randomized controlled trial. Nephrol Dial Transpl. 2020;35:274-82. 